\title{
Energy-minimizing two black holes initial data
}

\author{
Jacek Jezierski* \\ Katedra Metod Matematycznych Fizyki \\ Wydział Fizyki UW \\ ul. Hoża 69, Warszawa, Poland \\ Jerzy Kijowski† Szymon Łęski ${ }^{\ddagger}$ \\ Centrum Fizyki Teoretycznej PAN \\ Al. Lotników 32/46, Warszawa, Poland
}

October 25, 2018

\begin{abstract}
An attempt to construct the "ground state" vacuum initial data for the gravitational field surrounding two black holes is presented. The ground state is defined as the gravitational initial data minimizing the ADM mass within the class of data for which the masses of the holes and their distance are fixed. To parameterize different geometric arrangements of the two holes (and, therefore, their distance) we use an appropriately chosen scale factor. A method for analyzing the variations of the ADM mass and the masses (areas) of the horizons in terms of gravitational degrees of freedom is proposed. The Misner initial data are analyzed in this context: it is shown that they do not minimize the ADM mass.
\end{abstract}

\footnotetext{
${ }^{*}$ Partially supported by grant EPSRC: EP/D032091/1. Email: Jacek. Jezierski@fuw.edu.pl. ${ }^{\dagger}$ Email: kijowski@cft.edu.pl.

${ }^{\ddagger}$ Partially supported by the Foundation for Polish Science. Email: szleski@cft.edu.pl.
} 


\section{Introduction}

Equations of motion of charged point particles in classical electrodynamics can be derived from field equations within the following approach: a generic state of the composed "particles + field" system is treated as a perturbation of the "ground state" of the field, uniquely determined by the positions of the particles, see [1], [2]. In this classical approach the ground state is defined via a (non-local in time) decomposition of the actual field into the retarded (or advanced) field and the remaining "radiation field". Unfortunately, to decide what is the retarded or the advanced field, the entire trajectory of the particle must be known. Recently, it was shown that such a non-causal procedure may be avoided and the ground state may be defined via a conditional minimization of the (appropriately defined, "already renormalized") total energy of the "particles + field" system, with the positions and the charges of the particles being fixed (see e.g. 3], 44, [5]). Mathematically, this leads to a simple (elliptic) variational problem for the behaviour of the field in a topologically non-trivial region of $\mathbb{R}^{3}$ (exterior of the particles), where the charges of the particles provide the necessary boundary conditions. In this context, the ground state of the many particle system may be defined as the state of the field which contains ,,minimal amount of the radiation field", under condition that the charges and the positions of the particles are fixed.

There is a priori no obstruction against applying a similar idea to the problem of motion, and in particular to the two body problem, in General Relativity theory. Here, we have to replace point particles (elementary charges in electrodynamics) by black holes (elementary masses in General Relativity theory) and to consider perturbations of the field around a hypothetical "ground state" of the two black holes system. Such a ground state could be looked for as the state of the field, for which a conditional minimum of the total energy of the "black holes + gravitational radiation" system is achieved. Here, the total energy is equal to the ADM mass at infinity. This way we are led to a variational problem in a topologically non-trivial region of $\mathbb{R}^{3}$ (exterior of the two holes), where the variations must respect: 1) the Gauss-Codazzi constraints for the field data, 2) the masses of the holes (assigned to each of the horizons surrounding every hole and playing role of the boundary conditions) and, finally: 3) the fixed positions (distance) of the holes. The first two conditions are technically complicated but the last one is even more difficult to handle because it is ambiguous: the distance between the two black holes (horizons?) may be measured in many nonequivalent ways.

The idea of energy minimization is supported by the fact that, for a single black hole, the initial data for the Schwarzschild metric on the slice $t=$ const. correspond, indeed, to the minimum of the energy (ADM mass), with the area of the minimal surface surrounding it being fixed (see e.g. [6], [7], 8], [9]). Thus, the Schwarzschild initial data may be treated as a "ground state" of the one-body system. These data have vanishing extrinsic curvature and the induced three-metric is conformally flat. They lead to a static solution of Einstein equations.

In electrodynamics, the ground state of the many body system is always time symmetric. Indeed, any non-symmetricity means that the magnetic field does not vanish and 
increases the energy of the state. A similar argument (non-vanishing external curvature could only increase the total energy of the state), together with the example of the onebody problem (Schwarzschild) leads us to the conclusion that the gravitational two-body ground state should be looked for among time symmetric field configurations only. Such a conjecture is supported by the fact that critical points for the ADM mass correspond to the initial data generating stationary vacuum space-times (see e.g. [10], [11]).

Similarly as in electrodynamics, where there is no static solution for the two particles system, in General Relativity there is no static solution for two black holes (see [12]). The actual evolution of a hypothetical "two black holes + gravitational field" system state will surely display interaction between the ,,mechanical degrees of freedom" (described by positions and velocities of the holes) and the radiation degrees of freedom. We very much hope that the perturbation techniques based on the expansion with respect to the latter, in a phase-space neighbourhood of the ground state, could provide a good tool for the analysis of this evolution, at least in its initial phase. In the final stage of the evolution, treating the velocities on a perturbational level could become non-satisfactory. Then, a "dynamical ground state" would be necessary, where the time symmetry of the field configuration is dropped and where the velocities of the holes are encoded in the field boundary data on the horizons. This problem will be discussed in the future.

In the present paper we assume, therefore, vanishing extrinsic curvature of the field initial data in question, which also implies vanishing of the linear and angular momentum. This assumption simplifies the analysis of the constraints, vector constraints becoming trivial. What remains is the analysis of the scalar (Hamiltonian) constraint. On the other hand, we do not restrict ourselves to conformally flat metrics. Such an assumption, used by many authors, (see [13], [14], [15]), freezes all gravitational radiation degrees of freedom which are contained in the conformal part of the three-metric and - as shown in Section 6 - is incompatible with the minimization of the energy. Our strategy is to go beyond conformal flatness but keeping the same topology of the exterior of the two black holes. As a conclusion, we assume that initial data corresponding to the ground state we are looking for are: 1) time symmetric (no extrinsic curvature), 2) asymptotically flat, 3) the three-dimensional region $\Sigma$ where the field data are living is topologically $\Sigma=\mathbb{R}^{3} \backslash \mathcal{B}_{1} \backslash \mathcal{B}_{2}$ with two finite balls $\mathcal{B}_{1}$ and $\mathcal{B}_{2}$ being removed, and 4 ) the boundaries of these balls are minimal surfaces.

Our goal is to construct the (momentarily static) two black holes ground state as the vacuum initial data for gravitational field on $\Sigma$ (i.e. outside of the two minimal surfaces) for which the ADM mass (given by a boundary term at spatial infinity) is minimal within the set of three-metric tensors respecting: 1) the given masses of the holes and 2) their distance. Here, the following problems arise:

- How do we define the mass of each black hole? This can be done in many ways, e.g. using quasi-local mass approach (see [16]). Our choice is to assume that the mass of a black hole corresponds to the area of the minimal surface.

- How do we define the distance between black holes? Instead of e.g. geodesic distance, we propose to use a certain global parameter defined at spatial infinity, which seems 
to parameterize correctly the possible configurations of the two holes.

Our analysis of the scalar constraints enables us to construct an integral identity which relates the variation of the ADM mass with the changes of the three-metric in the volume and its behaviour at the boundary. When applied to the one black hole system, our procedure is strongly related to the proof of the Penrose inequality ([17]).

The integro-differential equations which result from our variational procedure are relatively complicated: there is little hope to be able to solve them analytically and to obtain an explicit formula for the gravitational ground state of the two black holes system. We hope, however, that an appropriate, numerical approximation will be useful for the description of the complete two body problem. In this paper we test our methods, applying them to simplified situations and show that this way we easily reproduce classical results: the Minkowski and Schwarzschild initial data are stationary points of the ADM mass (in the latter case the mass of the horizon must be fixed a priori). As a by-product of our method we show that the two black hole Misner data do not describe the minimum of the ADM mass.

The paper is organized as follows: in Section 2 we present our setup, proposed for description of the two-body data. We use a " $2+1$ foliation" which mimics the bispherical system of coordinates in the flat space $\mathbb{R}^{3}$ and we define unconstrained degrees of freedom which parameterize the admissible metrics. In the next Section we express the masses of the two interacting black holes and the total (ADM) mass of the "black holes + gravitational field" system in terms of the quantities defined in Section 2. In Section 4 we calculate variation of the ADM and the horizon masses in terms of the variation of the metric degrees of freedom. First, the calculation is performed for the conformally flat data and the result is applied to the analysis of the Schwarzschild (Section 5) and Misner (Section 6) data. Section 7 is the main part of the paper, where the above analysis is generalized to a generic (not necessarily conformally flat) case. In Section 8 we argue, using symmetry requirements, that the ground state of the two black hole system has only one non-trivial degree of freedom. Then, we analyze in detail this simplified (yet physically well-motivated) case. Finally, equations for the ground state are derived in Section 9. Technical details have been shifted to the Appendices.

\section{Conformal decomposition of the metric}

Consider a three-metric $g$ (a part of an initial data set for the gravitational field) on $\Sigma$, where $\Sigma$ is a three-dimensional manifold with boundary, which we obtain removing two finite balls $\mathcal{B}_{1}$ and $\mathcal{B}_{2}$ from $\mathbb{R}^{3}$. The boundary of $\Sigma$ consists of two disjoint connected (spherical) components $H_{i}=\partial \mathcal{B}_{i}: \partial \Sigma=H=H_{1} \cup H_{2}$. We assume $g$ to be a Riemannian metric on $\Sigma$, asymptotically flat in the following sense:

$$
g=\Psi^{4} \mathbf{g}, \quad \mathbf{g}=\mathbf{b}+\rho,
$$

where the conformal factor $\Psi$ is a positive function on $\Sigma, \mathbf{b}$ is a flat three-metric and we impose appropriate decay conditions for the metric $g$, namely $\rho=O\left(1 / r^{2}\right), \partial \rho=O\left(1 / r^{3}\right)$, 
$\Psi=O(1)$ and $\partial \Psi=O\left(1 / r^{2}\right)$. We show in the sequel (cf. Section 9 and Appendix D) that these decay conditions are compatible with the ground-state problem.

We are going to use on $\Sigma$ a "bi-spherical" system of coordinates. More precisely, we parameterize the one-point compactification of $\Sigma$ by the cylinder

$$
\Sigma \cup\{\infty\} \simeq S^{2} \times I:=W,
$$

where $S^{2}$ is the topological two-sphere and $I$ is an interval $[-a, b] \subset \mathbb{R}$.

On $W$ we introduce coordinates $x^{i}, i=1,2,3$, adapted to the foliation, i.e. $\mu=$ $x^{3} \in[-a, b]$, and $x^{A}, A=1,2$, are spherical coordinates $(\eta, \varphi)$ on each of the spheres $\mu=$ const. We assume that $H_{1}\left(H_{2}\right.$, respectively) correspond to the value $\mu=-a(\mu=b$, respectively) and that infinity corresponds to the north pole $(\eta=0)$ on the sphere $\mu=0$.

Such parameterizations are subject to a three-parameter (per point) diffeomorphism group. To choose a specific one amongst them, three gauge conditions have to be imposed. To fix coordinates $(\eta, \varphi)$ on each sphere $\{\mu=$ const. $\}$, we assume that the two-dimensional part of the metric $g$ (and, therefore, also of the metric $\mathbf{g}$ ) is proportional to the standard, unit, round metric on $S^{2}$ :

$$
\sigma_{A B} \mathrm{~d} x^{A} \mathrm{~d} x^{B}=\mathrm{d} \eta^{2}+\sin ^{2} \eta \mathrm{d} \varphi^{2} .
$$

The above (two per point) gauge conditions give rise to the further conformal decomposition of the metric $\mathrm{g}$ :

$$
\mathbf{g}=h^{2} w
$$

where $w$ is a metric on the cylinder $W:=S^{2} \times I$, such that its restriction to every leave coincides with (2): $w_{A B}=\sigma_{A B}$. The entire information about such a metric is contained, therefore, in the following vector-density:

$$
D^{k}:=\sqrt{\operatorname{det} g_{A B}} g^{3 k}=\sigma w^{3 k},
$$

where $w^{i j}$ and $g^{i j}$ are the inverse (contravariant) metric tensors and $\sigma=\sqrt{\operatorname{det} \sigma_{A B}}=\sin \eta$. The remaining gauge condition, fixing the coordinate $\mu$ (and, therefore, leaves of the foliation $\{\mu=$ const. $\}$ ), may be expressed in terms of a differential equation imposed on the "electric field" $D$. In previous works [6], [7], [18, [19], conditions such as $\partial_{k} D^{k}=0$ were successfully used in topologically different arrangements. Most results of this paper do not rely on a specific choice of the gauge. As will be seen in Section 8 , some results simplify considerably if we assume vanishing of the mean external curvature of the leaves $\{\mu=$ const. $\}$, calculated with respect to the metric $w$ (i.e. condition $k_{w}=0$ ). The applicability of this condition in a generic situation needs further investigation.

Having at our disposal the product of two conformal factors: $\Psi$ and $h$, we are free to fix arbitrarily one of them. We choose the standard value:

$$
h:=\frac{1}{\cosh \mu-\cos \eta},
$$


which relates the flat, Euclidean three-metric $\mathbf{b}$ on $\mathbb{R}^{3}$, to the cylindrical metric $\stackrel{\circ}{w}=$ $\mathrm{d} \mu^{2}+\mathrm{d} \eta^{2}+\sin ^{2} \eta \mathrm{d} \varphi^{2}$ on $W$ :

$$
\mathbf{b}=\mathrm{d} x^{2}+\mathrm{d} y^{2}+\mathrm{d} z^{2}=\frac{1}{(\cosh \mu-\cos \eta)^{2}}\left[\mathrm{~d} \mu^{2}+\mathrm{d} \eta^{2}+\sin ^{2} \eta \mathrm{d} \varphi^{2}\right]=h^{2} \stackrel{\circ}{w}
$$

via the bispherical coordinates $(\mu, \eta, \varphi)$ :

$$
\begin{aligned}
x & =\cos \varphi \frac{\sin \eta}{\cosh \mu-\cos \eta}, \\
y & =\sin \varphi \frac{\sin \eta}{\cosh \mu-\cos \eta}, \\
z & =\frac{\sinh \mu}{\cosh \mu-\cos \eta} .
\end{aligned}
$$

The quantity $D$ takes in this case the following value:

$$
\stackrel{\circ}{D}^{3}=\sigma, \quad \stackrel{\circ}{D}^{A}=0 .
$$

We shall use in parallel both of the two conformal decompositions of the metric $g$ defined above. Putting

$$
\Phi:=\Psi \sqrt{h}
$$

we have:

$$
g=\Phi^{4} w=\Psi^{4} \mathbf{g}
$$

and the following (rescaling) transformation law for the Ricci scalar holds:

$$
\Phi^{4} \mathcal{R}(g)=\mathcal{R}(w)-\frac{8}{\Phi} \Delta_{w} \Phi
$$

where by $\Delta_{w}$ we denote Laplace-Beltrami operator associated with the metric $w$. The above formula implies the following equations satisfied by the conformal factors $\Phi$ and $\Psi$ :

$$
\begin{aligned}
& \sqrt{\operatorname{det} w} \Phi\left(\Delta_{w}-\frac{\mathcal{R}(w)}{8}\right) \Phi=-\frac{1}{8} \sqrt{\operatorname{det} g} \mathcal{R}(g), \\
& \sqrt{\operatorname{det} \mathbf{g} \Psi}\left(\Delta_{\mathbf{g}}-\frac{\mathcal{R}(\mathbf{g})}{8}\right) \Psi=-\frac{1}{8} \sqrt{\operatorname{det} g} \mathcal{R}(g) .
\end{aligned}
$$

For the special case $w=\stackrel{\wp}{w}$ and $\Phi=\sqrt{h}=1 / \sqrt{\cosh \mu-\cos \eta}$ we have: $g=\mathbf{b}, \mathcal{R}(\stackrel{w}{*})=2$, $\mathcal{R}(g)=0$ and equation (9) takes the form:

$$
\left(\Delta_{\grave{w}}-\frac{1}{4}\right) \sqrt{h}=0 .
$$

However, because of singularity of the function $\sqrt{h}$ at the point $\mu=0, \eta=0$ (i.e. at infinity), this condition is fulfilled outside of this point, only. It is easy to check that, globally, the following distributional equation is satisfied, instead of (11):

$$
\left(\Delta_{\grave{w}}-\frac{1}{4}\right) \sqrt{h}=-4 \pi \boldsymbol{\delta}_{0} .
$$


This means that for any (smooth) test function $f \in C_{0}^{\infty}(W)$ we have:

$$
\int_{W} \Phi\left(\Delta_{\grave{w}} f-\frac{1}{4} f\right) \sqrt{\operatorname{det} \stackrel{o}{w}}=-4 \pi f(\mu=0, \eta=0) .
$$

The Dirac delta distribution here reflects the fact that a small sphere surrounding $\mu=0$, $\eta=0$ maps to a large sphere in the metric $\mathbf{b}$. In the limit, point $\mu=0, \eta=0$ corresponds to the sphere at infinity $S_{\infty}$.

The two ends of this cylinder, obtained for $\mu \rightarrow \pm \infty$, correspond to the points $(x, y, z)=(0,0, \pm 1)$ in the flat, Euclidean three-space. We can interpret these points as two test bodies in the flat-metric limit. The distance between these two points is standard and equals 2. To allow arbitrary distances, there are two possible methods: 1) to change transformation laws (5)-(7), or 2) to allow an extra multiplicative factor $d \in \mathbb{R}_{+}$ in formula (4). In this paper we are going to use always the last option, which implies also an extra multiplicative factor $\sqrt{d}$ at the right-hand side of equation (12). Then $g=d \cdot \mathbf{b}$ and the distance between endpoints $\mu=\infty$ and $\mu=-\infty$ equals $2 d$. The parameter $d$ may be thought of as a "scale factor". On the other hand, the position of the interacting heavy bodies (black holes) in the coordinate space will always be standardized.

In a generic (not necessarily flat) case, the scalar constraint $\mathcal{R}(g)=0$, together with geometric identities (9) and (10), imply the following equations:

$$
\begin{gathered}
\left(\Delta_{\mathbf{g}}-\frac{\mathcal{R}(\mathbf{g})}{8}\right) \Psi=0, \\
\left(\Delta_{w}-\frac{\mathcal{R}(w)}{8}\right) \Phi=-4 \pi \sqrt{d} \boldsymbol{\delta}_{0},
\end{gathered}
$$

where, again, we have admitted an arbitrary scale factor $\sqrt{d}=\left.\Psi\right|_{\infty}$ on the right-hand side. (Note that $w \rightarrow \stackrel{w}{w}$ for $\mu, \eta \rightarrow 0$. Hence, we may use either $\sqrt{\operatorname{det} w}$ or $\sqrt{\operatorname{det} \stackrel{\circ}{w}}$ in the integral (13).) In the sequel, we are going to interpret $2 d$ as a quantity parameterizing the distance between two black holes, even beyond the limit of point-like bodies in flat space. It was already proved [20] that in the limit $d \rightarrow \infty$ of the Misner data, the value $2 d$ gives, indeed, the exact distance between the bodies.

For our purposes, however, it is sufficient to treat $d$ as a scale factor: its rescaling by a multiplicative factor implies rescaling of all the distances appearing in our problem. In any specific geometric situation the value of $d$ is defined uniquely once a specific gauge condition for the coordinate $\mu$ is chosen.

In a generic case of two black holes we constrain $\mu$ to a finite interval $[-a, b]$. Given "independent degrees of freedom" of the gravitational field, described by $D^{k}$ (i.e. by the metric $w$ ), we want to retrieve the remaining information about the physical metric $g$ from equation (15) on $W$. The Dirac delta and the scale factor $d$ in (15) play role of "boundary conditions at infinity". The remaining boundary conditions on the spheres $H_{1}:=\{\mu=-a\}$ and $H_{2}:=\{\mu=b\}$ are implied by the fact that we want these twosurfaces to be horizons. We assume, therefore, that they are minimal in the ambient 
three-metric $g$, i.e. that their mean extrinsic curvature $k$ vanishes: $k=0$. We use the following law of transformation of $k$ under conformal rescaling:

$$
\Phi^{3} k=\Phi k_{w}-4 \nabla_{w}^{\perp} \Phi
$$

where $k_{w}$ is the curvature calculated with respect to metric $w$, and $\nabla_{w}^{\perp}$ is a projection (with respect to $w$ ) of the gradient on the unit (again in metric $w$ ), outward normal vector. This yields

$$
\left.k\right|_{H_{i}}=\left.0 \Longleftrightarrow \nabla \frac{\perp}{w} \Phi\right|_{H_{i}}=\left.\frac{\Phi k_{w}}{4}\right|_{H_{i}} .
$$

In particular, if $k_{w}=0$, we get the Neumann boundary conditions for $\Phi$; for a particular, flat case $w=\stackrel{w}{w}$, we have

$$
\left.k\right|_{H_{i}}=\left.0 \Longleftrightarrow \frac{\partial \Phi}{\partial \mu}\right|_{H_{i}}=0 .
$$

For the conformal factor $\Psi$ these conditions may be written as follows: (compare [21, equation (36)]):

$$
\left.\Psi\right|_{\infty}=\sqrt{d},\left.\quad\left(\frac{\partial}{\partial \mu} \Psi+\Psi \frac{\partial}{\partial \mu}(\log \sqrt{h})\right)\right|_{H_{i}}=0 .
$$

As an example of the two-body data fitting into the above framework we may take the Misner metric [14] or its generalization [22], see [20] for an explicit formula. The metric is of the form:

$$
g=\Psi_{m}^{4} \mathbf{b}
$$

and there are two minimal surfaces surrounding the singularities of the conformal factor. The minimal surfaces are metric spheres in the conformal metric $\mathbf{b}$, hence it is natural to use the $2+1$ foliation given by bispherical coordinates; the minimal surfaces correspond to $\mu= \pm \mu_{0}$ (or, to $\mu=-a, \mu=b$ in case of non-equal masses). The conformal metric $\mathbf{b}$ is then rewritten in terms of the cylindric metric (44). On the other hand, the Brill and Lindquist data, [15], are more difficult to handle within this framework. This is due to the fact that the minimal surfaces are not round spheres in the conformal metric and, whence, we can not use the standard bispherical coordinates to construct the foliation. However, it is always possible to construct a topologically bispherical foliation for these data, for which the metric $w$ is no longer equal to $\stackrel{\circ}{w}$.

\section{ADM mass and masses of the horizons}

For time-symmetric initial data the Hamiltonian constraints reduces to $\mathcal{R}(g)=0$. Equation (10) can, therefore, be written as:

$$
-8 \sqrt{\operatorname{det} \mathbf{g}} \nabla_{\mathbf{g}}\left(\Psi \nabla_{\mathbf{g}} \Psi\right)=-8 \sqrt{\operatorname{det} \mathbf{g}}\left|\nabla_{\mathbf{g}} \Psi\right|^{2}-\Psi^{2} \sqrt{\operatorname{det} \mathbf{g}} \mathcal{R}(\mathbf{g}),
$$


where $\left|\nabla_{\mathbf{g}} \Psi\right|^{2}$ is the length of the gradient of $\Psi$ with respect to $\mathbf{g}$. Let us integrate formula (20) over the whole space $\Sigma$. The complete divergence on the left-hand side of equation (20) yields a boundary integral. Hence we get:

$$
\begin{array}{r}
-8 \lim _{r \rightarrow \infty} \int_{S_{r}} \Psi \nabla_{\mathbf{g}}^{\perp} \Psi \sqrt{\left.\operatorname{det} \mathbf{g}\right|_{S_{r}}}-8 \int_{H} \Psi \nabla_{\mathbf{g}}^{\perp} \Psi \sqrt{\left.\operatorname{det} \mathbf{g}\right|_{H}} \\
=-\int_{\Sigma}\left(8\left|\nabla_{\mathbf{g}} \Psi\right|^{2}+\Psi^{2} \mathcal{R}(\mathbf{g})\right) \sqrt{\operatorname{det} \mathbf{g}}
\end{array}
$$

where by $\nabla_{\mathbf{g}}^{\perp}$ we denote the projection (with respect to $\mathbf{g}$ ) of the gradient on the unit, outward, normal vector (analogous to $\nabla_{w}^{\perp}$ ); $S_{r}$ are spheres of radius $r$ (in metric b). By $\left.\mathrm{g}\right|_{S}$ we denote the pullback of the metric to $S$, i.e. the induced metric. We are going to show in the sequel that the first of the integrals yields the ADM mass:

$$
16 \pi m_{A D M}=-8 \lim _{r \rightarrow \infty} \int_{S_{r}} \Psi \nabla_{\mathbf{g}}^{\perp} \Psi \sqrt{\left.\operatorname{det} \mathbf{g}\right|_{S_{r}}} .
$$

For this purpose consider a three-metric $g$ of ADM mass $m$ and let it satisfy the decay conditions we have imposed. Then, for $d=1,\left.\Psi\right|_{\infty}=\sqrt{d}=1$, the metric $g$ has the following form in terms of the asymptotic spherical coordinates $(r, \theta, \varphi)$ :

$$
g=\left(1+\frac{m}{2 r}+O\left(\frac{1}{r^{2}}\right)\right)^{4}\left(\mathrm{~d} r^{2}+r^{2} \mathrm{~d} \vartheta^{2}+r^{2} \sin ^{2} \vartheta \mathrm{d} \varphi^{2}+O\left(\frac{1}{r^{2}}\right)\right)
$$

Using the above form of the metric, formula (22) may be checked by inspection. Rescaling of $r$ by an arbitrary factor $d$, i.e. using the Ansatz $r=\widetilde{r} d$, leads to an arbitrary value $\left.\Psi\right|_{\infty}=\sqrt{d}$ and to the following, asymptotic form of the metric tensor:

$$
g=\left(\sqrt{d}+\frac{m}{2 \sqrt{d} \widetilde{r}}+O\left(\frac{1}{\widetilde{r}^{2}}\right)\right)^{4}\left(\mathrm{~d} \widetilde{r}^{2}+\widetilde{r}^{2} \mathrm{~d} \vartheta^{2}+\widetilde{r}^{2} \sin ^{2} \vartheta \mathrm{d} \varphi^{2}+O\left(\frac{1}{\widetilde{r}^{2}}\right)\right)
$$

Simple calculations show that equation (22) still holds.

Using the relation between $\Phi$ and $\Psi$ we can express the ADM mass in terms of $\Phi$ :

$$
16 \pi m_{A D M}=-8 \lim _{\varepsilon \rightarrow 0} \int_{S_{\varepsilon}}\left[\Phi \nabla_{w}^{\perp} \Phi-\left(\frac{\Phi}{\sqrt{h}}\right)^{2} \sqrt{h} \nabla_{w}^{\perp} \sqrt{h}\right] \sqrt{\left.\operatorname{det} w\right|_{S_{\varepsilon}}},
$$

where we subtract the renormalization term $\sqrt{h} \nabla \sqrt{h}$ (corresponding to the flat metric) from the term $\Phi \nabla \Phi$. We integrate over surfaces $\varepsilon=$ const., where $\varepsilon^{2}=\mu^{2}+\eta^{2}$.

We define the mass of a black hole in terms of the area of the minimal surface surrounding it1:

$$
m_{H_{i}}=\sqrt{\frac{\int_{H_{i}} \lambda}{16 \pi}}
$$

\footnotetext{
${ }^{1}$ The energy $m_{H_{i}}$ plays role of the lower bound in Penrose inequality (cf. [17, [23]) and never decreases according to the second law of black hole physics (see e.g. 24] or [25]).
} 
where by $\lambda$ we denote the two-dimensional volume element on the leaves $\{\mu=$ const. $\}$ : $\lambda:=\sqrt{\operatorname{det} g_{A B}}$.

Given a solution $\Phi$ of equation (15) with boundary conditions (18) (or, equivalently, a solution $\Psi$ of equation (14) with boundary conditions (19)) the areas of the minimal surfaces (i.e. the masses of black holes) are given. We have, therefore, an indirect control over these masses by an appropriate choice of $a$ and $b$.

\section{Variations of conformally flat initial data}

We are going to prove in the sequel that the ground state of two interacting black holes cannot be described by conformally flat data. The search of an appropriate ground state must go, therefore, beyond conformal flatness. For this purpose consider a perturbation of the conformally flat metric. Denote

$$
g=\Phi^{4} w, \quad \stackrel{\circ}{g}=\stackrel{\circ}{\Phi}^{4} \stackrel{\circ}{w},
$$

and decompose the degrees of freedom of the metric $w$ as deformations of the degrees of freedom of $\stackrel{\circ}{w}: D^{k}:=\stackrel{\circ}{D}^{k}+\delta D^{k}$, where $\stackrel{\circ}{D}$ is given by equation (8). Using formula

$$
\Delta_{g} \Phi=\frac{1}{\sqrt{g}} \partial_{i}\left(g^{i j} \sqrt{g} \partial_{j} \Phi\right)
$$

we have the following linearization of the above quantity:

$$
\Delta_{g} \Phi=\Delta_{\grave{g}} \delta \Phi+\left(1-\frac{1}{\sqrt{g}} \delta \sqrt{g}\right) \Delta_{g}^{\circ} \stackrel{\circ}{\Phi}+\frac{1}{\sqrt{g}} \partial_{i}\left(\delta \sqrt{g} \stackrel{\circ}{g}^{i j} \partial_{j} \stackrel{\circ}{\Phi}+\sqrt{\stackrel{\circ}{g}} \delta g^{i j} \partial_{j} \stackrel{\circ}{\Phi}\right),
$$

and, consequently, the following equation for $\delta \Phi$ :

$$
\begin{aligned}
\left(\Delta_{\mathscr{w}}-\frac{1}{4}\right) \delta \Phi= & \frac{1}{8} \delta \mathcal{R} \stackrel{\circ}{\Phi}-\frac{1}{2} \frac{\delta D^{3}}{\sigma} \Delta_{\dot{\omega}} \stackrel{\circ}{\Phi} \\
& -\frac{1}{\sigma} \partial_{i}\left[\left(\sigma \delta w^{i j}-\frac{1}{2} \delta D^{3} \stackrel{\circ}{w}^{i j}\right) \partial_{j} \stackrel{\circ}{\Phi}\right] .
\end{aligned}
$$

We denote the right-hand side of this equation by $j[w]$ :

$$
j[w]=\frac{1}{8} \delta \mathcal{R}(w) \stackrel{\circ}{\Phi}-\frac{1}{2} \frac{\delta D^{3}}{\sigma} \Delta_{\stackrel{\circ}{\circ}} \stackrel{\circ}{\Phi}-\frac{1}{\sigma} \partial_{i}\left[\left(\sigma \delta w^{i j}-\frac{1}{2} \delta D^{3} \stackrel{\circ}{w}^{i j}\right) \partial_{j} \stackrel{\circ}{\Phi}\right] .
$$

Moreover, we keep the scale factor unchanged $(\delta d=0)$ and, whence, the right-hand side of the linearized equation (15) vanishes. This is why the right-hand side of (29) contains no Dirac delta term. The boundary condition for $\delta \Phi$ are such that $H_{i}$ remain minimal surfaces:

$$
\left.\nabla \frac{\perp}{\grave{w}} \delta \Phi\right|_{H_{i}}=\left.\frac{\partial(\delta \Phi)}{\partial \mu}\right|_{H_{i}}=0 .
$$


The formula (22) for the ADM mass can also be linearized around the metric $\stackrel{\circ}{\mathrm{g}}=h^{2} \stackrel{\circ}{\mathrm{w}}$ :

$$
16 \pi \delta m_{A D M}=-8 \lim _{r \rightarrow \infty} \int_{S_{r}}\left(\stackrel{\circ}{\Psi} \nabla_{\grave{\omega}}^{\perp} \delta \Psi+\delta \Psi \nabla_{\dot{w}}^{\perp} \stackrel{\circ}{\Psi}\right) \sqrt{\left.\operatorname{det} \stackrel{\circ}{\mathrm{g}}\right|_{S_{r}}} .
$$

Because the contribution of the second term under the integral vanishes, the formula may be rewritten as follows:

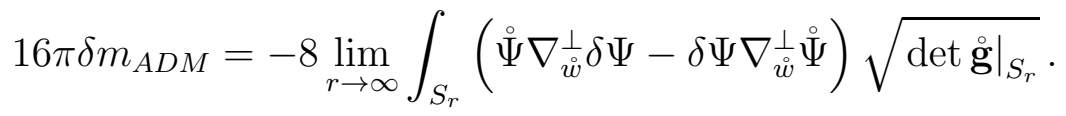

In fact, we have $\delta \Psi \rightarrow 0$ at infinity (because the scale factor is kept unchanged: $\delta d=0$ ) and $\nabla_{\dot{w}}^{\perp} \stackrel{\circ}{\Psi}=O\left(\frac{1}{r^{2}}\right)$, which proves (33). Rewriting it in terms of $\delta \Phi$ we obtain:

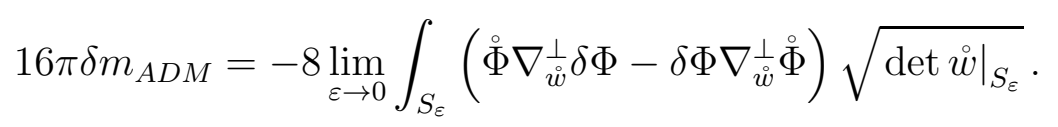

We are going to express the above variation of the ADM mass in terms of variations $\delta D^{k}$ of gravitational degrees of freedom. Observe that, due to elliptic equation (29), the variation $\delta \Phi$ of the conformal factor depends non-locally upon variations $\delta D^{k}$. To handle this non-local dependence, it is useful to rewrite the surface integral (34) in term of a volume integral. Next, we shall transform the expression in such way that the dependence upon $\delta D^{k}$ becomes explicit. For this purpose we rewrite equations (29) and (9) in the following form:

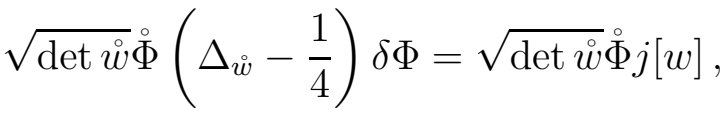

$$
\begin{aligned}
& \sqrt{\operatorname{det} \stackrel{\circ}{\omega}} \Phi\left(\Delta_{\grave{w}}-\frac{1}{4}\right) \stackrel{\circ}{\Phi}=0 .
\end{aligned}
$$

Subtracting these equations we get:

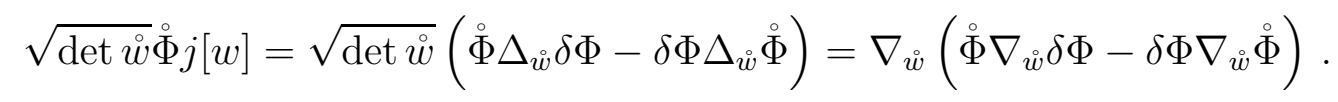

If we multiply the above divergence by -8 and integrate it over $W^{*}:=W \backslash\{(\mu=0, \eta=0)\}$ then the boundary term at infinity reproduces precisely formula (34). Finally, we obtain:

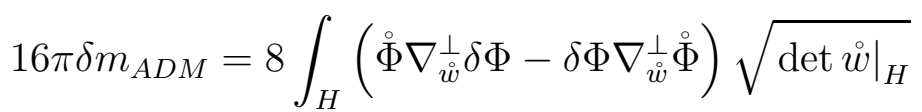

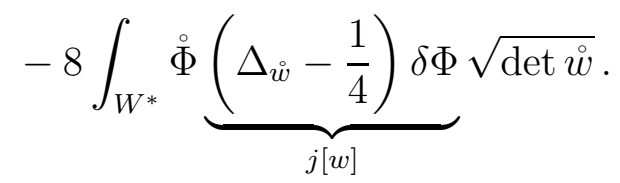

The first integral in (38) vanishes because of the boundary conditions: (18) for $\stackrel{\circ}{\Phi}$ and (31) for $\delta \Phi$. Consequently, we have:

$$
16 \pi \delta m_{A D M}=-8 \int_{W^{*}} \stackrel{\circ}{\Phi} j[w] \sqrt{\operatorname{det} \stackrel{\circ}{w}} .
$$


In the next step we rewrite variations $\delta \mathcal{R}$ and $\delta w^{i j}$ in $j[w]$ (see equation (30)) in terms of $\delta D^{k}$. (Detailed calculations for $\delta \mathcal{R}$ have been shifted to Appendix A.) This way integral (39) may be rewritten as an expression containing variations $\delta D^{k}$ and their derivatives. In the last step we eliminate the latter using integration by parts (boundary integral vanishes because we assume that $\delta D^{k}$ vanish at the boundary). It is convenient to formulate the final result in terms of the following covector-valued, symmetric, bilinear form $B_{k}(f, g)$ :

$$
\begin{gathered}
B_{A}(f, g)=-\frac{1}{4}(f g)_{, 3 A}+2 f_{(, A} g_{, 3)}, \\
B_{3}(f, g)=\frac{1}{8}(\stackrel{2}{\Delta}-1)(f g)-\frac{1}{2} \sigma^{A B} f_{, A} g_{, B}+\frac{1}{2} f_{, 3} g_{, 3},
\end{gathered}
$$

where by $\stackrel{2}{\Delta}$ we denote the Laplace-Beltrami operator on the unit sphere (in metric $\sigma$ ). Then the following formula holds for an arbitrary function $f$ :

$$
\int_{W^{*}} f j[w] \sqrt{\stackrel{\circ}{w}}=\int_{W^{*}} B_{k}(f, \stackrel{\circ}{\Phi}) \delta D^{k} .
$$

In particular, putting $f=\stackrel{\circ}{\Phi}$, we may rewrite formula (39) as follows:

$$
16 \pi \delta m_{A D M}=-8 \int_{W^{*}} B_{k}(\stackrel{\circ}{\Phi}, \stackrel{\circ}{\Phi}) \delta D^{k} .
$$

We want to restrict the above variation to the class of those $\delta D^{k}$ 's, for which the masses $m_{H_{i}}, i=1,2$, of both our black holes remain unchanged. For this purpose we are going to use the Lagrange multipliers method as soon as we are able to express the variations $\delta m_{H_{i}}$ in terms of appropriate volume integrals. We begin with the following formula:

$$
16 \pi \delta m_{H_{i}}=\frac{1}{2 \stackrel{\circ}{m}_{H_{i}}} \int_{H_{i}} \delta \lambda=\frac{1}{2 \stackrel{\circ}{m}_{H_{i}}} \int_{H_{i}} 4 \stackrel{\circ}{\Phi}^{3} \delta \Phi \sigma .
$$

Denote by $\widehat{m}_{H_{i}}$ the (unique) function satisfying equation

$$
\left(\Delta_{\tilde{w}}-\frac{1}{4}\right) \widehat{m}_{H_{i}}=0
$$

and boundary conditions

$$
\left.\nabla \stackrel{\perp}{\stackrel{\perp}{w}} \widehat{m}_{H_{i}}\right|_{H_{j}}=\frac{1}{4} \frac{\stackrel{\circ}{\Phi^{3}}}{\stackrel{\circ}{m}_{H_{i}}} \delta_{i j},
$$

where $\delta_{i j}$ is the Kronecker symbol. Due to condition: $\left.\nabla \frac{\perp}{\tilde{w}} \delta \Phi\right|_{H_{i}}=0$, integration by parts leads to the formula:

$$
\begin{aligned}
16 \pi \delta m_{H_{i}} & =-8 \int_{H_{i}}\left(\widehat{m}_{H_{i}} \nabla_{\stackrel{\perp}{\omega}}^{\perp} \delta \Phi-\delta \Phi \nabla_{\dot{w}}^{\perp} \widehat{m}_{H_{i}}\right) \sigma \\
& =-8 \int_{W^{*}} \widehat{m}_{H_{i}}\left(\Delta_{\dot{w}}-\frac{1}{4}\right) \delta \Phi \sigma
\end{aligned}
$$


the last equality being true because the boundary term vanishes at infinity. Hence, both quantities: $\delta m_{A D M}$ (cf. (43)) and $\delta m_{H_{i}}$, may be expressed by similar volume integrals of the form $f\left(\Delta_{\tilde{w}}-\frac{1}{4}\right) \delta \Phi$, where $f$ is a solution to, respectively:

$$
\left(\Delta_{\check{w}}-\frac{1}{4}\right) f= \begin{cases}0 & \text { for } m_{H_{i}} \\ -4 \pi \boldsymbol{\delta}_{0} \cdot \sqrt{d} & \text { for } m_{A D M}\end{cases}
$$

with appropriately chosen boundary conditions. Such $f$ will be called respectively the ADM mass increase factor or the horizon mass increase factor. We already know that the conformal factor is the ADM mass increase factor: $\widehat{m}_{A D M}=\stackrel{\circ}{\Phi}$. Hence, we have

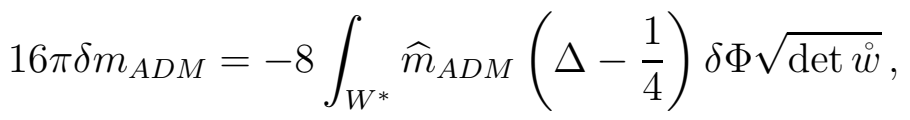

$$
\begin{aligned}
& 16 \pi \delta m_{H_{i}}=-8 \int_{W^{*}} \widehat{m}_{H_{i}}\left(\Delta-\frac{1}{4}\right) \delta \Phi \sqrt{\operatorname{det} \stackrel{\leftrightarrow}{w}} .
\end{aligned}
$$

A field configuration minimizing the ADM mass within the class of data with fixed masses of black holes must, therefore, annihilate the following form:

$$
\begin{aligned}
16 \pi\left(\delta m_{A D M}-\sum_{i} \nu_{i} \delta m_{H_{i}}\right) & =-8 \int_{W^{*}}\left(B_{k}(\stackrel{\circ}{\Phi}, \stackrel{\circ}{\Phi})-\sum_{i} \nu_{i} B_{k}\left(\widehat{m}_{H_{i}}, \stackrel{\circ}{\Phi}\right)\right) \delta D^{k} \\
& =-8 \int_{W^{*}} B_{k}\left(\left(\stackrel{\circ}{\Phi}-\sum_{i} \nu_{i} \widehat{m}_{H_{i}}\right), \stackrel{\circ}{\Phi}\right) \delta D^{k},
\end{aligned}
$$

where $\nu_{i}$ are Lagrange multipliers. Observe that, moreover, the above variation procedure respects the scale factor $d$ which remains unchanged. We conclude that vanishing of the right-hand side for an arbitrary variation $\delta D^{k}$ is necessary if our field configuration has to realize the conditional minimum of the total energy of the "two black holes + gravitational field" system, where the masses of the holes and their distance are fixed 2 .

\section{$5 \quad$ Stability of the Schwarzschild initial data}

To test our method we will apply the above formulae to the flat metric $\mathbf{b}$ and the Schwarzschild metric $g_{s}$. The flat metric

$$
\mathbf{b}=d^{2} h^{2} \stackrel{\circ}{ }
$$

describes the field configuration surrounding two "zero-mass black holes", i.e. two arbitrarily chosen points of the flat space. We have: $B_{k}(\sqrt{h}, \sqrt{h})=0$. This corresponds in our approach to the (weak) stability of the Minkowski initial data [26].

\footnotetext{
${ }^{2}$ In fact, only two variations among the three $\delta D^{k}$ 's represent the change of the field configuration, whereas the third one represents variation of a gauge condition - at this point not yet fixed.
} 
The Schwarzschild metric in the bispherical setting may be written as follows (cf. formula (24)):

$$
g_{s}=\Phi_{s}^{4} \stackrel{\circ}{w}=\left(\sqrt{h} \sqrt{d}+\frac{m}{2 \sqrt{d}} \frac{\sqrt{h}}{\bar{r}}\right)^{4} \stackrel{\leftrightarrow}{w} .
$$

Here $m$ is the mass of the unique real horizon, whereas $2 d$ parameterizes its "distance" from an arbitrarily chosen fictitious "zero-mass black hole". The radius $\bar{r}$ is a function of $(\mu, \eta, \varphi)$ and parameters $m$ and $d$ (see Appendix B for details). In this case we have: $B_{k}\left(\Phi_{s}, \Phi_{s}\right) \neq 0$, because a generic deformation $\delta D^{k}$ changes the mass of the hole. To calculate the right-hand side of (51) we use an explicit expression for the $\widehat{m}_{H}$ factor:

$$
\widehat{m}_{H}=\frac{m}{\sqrt{d}} \frac{\sqrt{h}}{\bar{r}},
$$

which may be easily verified. On the other hand, we have:

$$
\Phi_{s}=\sqrt{d} \sqrt{h}+\frac{m}{2 \sqrt{d}} \frac{\sqrt{h}}{\bar{r}},
$$

and, consequently:

$$
\Phi_{s}=\sqrt{d} \sqrt{h}+\hat{f}, \quad \widehat{m}_{H}=2 \hat{f} .
$$

The form $B_{k}(f, g)$ is bilinear and symmetric, hence we get

$$
\begin{aligned}
& B_{k}\left(\Phi_{s}, \Phi_{s}\right)-\nu B_{k}\left(\widehat{m}_{H}, \Phi_{s}\right)= \\
& \quad d \cdot B_{k}(\sqrt{h}, \sqrt{h})+(1-2 \nu) B_{k}(\hat{f}, \hat{f})+(2-2 \nu) \sqrt{d} B_{k}(\sqrt{h}, \hat{f}),
\end{aligned}
$$

where, similarly as in the flat case, we have $B_{k}(\sqrt{h}, \sqrt{h})=0$. It is also easy to check that $B_{k}(\hat{f}, \hat{f})=0$. Hence, the above quantity vanishes for $\nu=1$. The interpretation of this result is following: on a class of $D^{k}$ 's with appropriate asymptotics, a map $D^{k} \mapsto m_{A D M}\left[D^{k}\right]$ may be defined. Constraining the map to those $D^{k}$, for which $m_{H}$ remains unchanged, we see that the linear part of $m_{A D M}\left[D^{k}\right]$ remains unchanged, i.e. the Schwarzschild metric is a stationary point of this map. In other words, a small deformation of the Schwarzschild metric which does not change the area of the horizon will not change the total ADM mass (cf. [6], [27]).

\section{Application to Misner data}

The two-body data, which can be easily analyzed using our method, have been proposed

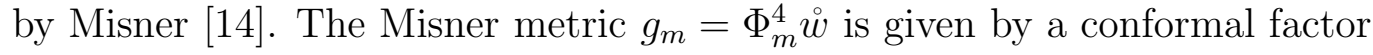

$$
\Phi_{m}=\sum_{n \in \mathbb{Z}} \frac{\sqrt{d}}{\sqrt{\cosh \left(\mu+2 n \mu_{0}\right)-\cos \eta}}
$$


defined on $S^{2} \times\left[-\mu_{0}, \mu_{0}\right]$. The spheres $\mu= \pm \mu_{0}$ are minimal surfaces of equal mass $m=m\left(\mu_{0}, d\right)$. The above definition may be generalized to the case of non-equal masses [22], the explicit formulae for $\Phi$ are given in [20]. According to (8) we have $\stackrel{\circ}{ }^{3}=\sigma$, $\stackrel{D}{ }^{A}=0$.

Our method of variation allows us to prove that, using a small perturbation of the metric, one can decrease the ADM mass of the above field configurations, without changing the masses of both horizons and the scale factor $d$. (See [28, 29] for previous results obtained using different techniques.) To show this we use the fact that only two of three degrees of freedom $D^{k}$ are independent: an additional gauge condition fixing the foliation $\{\mu=$ const. $\}$ may be imposed. We choose the following gauge condition:

$$
k_{w}=0
$$

In this gauge we have

$$
k_{w}=\left(\frac{\sigma D^{A}}{D^{3}}\right)_{, A}=0,
$$

hence, there is a function $\chi$ such that we have $\left(\varepsilon^{A B}\right.$ is the two-dimensional Levi-Cività symbol):

$$
\delta \frac{\sigma D^{A}}{D^{3}}=\varepsilon^{A B} \chi_{, B}
$$

We have also

$$
B_{3} \delta D^{3}+B_{A} \delta D^{A}=B_{3} \delta D^{3}+\frac{\stackrel{\circ}{D}^{3}}{\sigma} B_{A} \delta\left(\frac{\sigma D^{A}}{D^{3}}\right)+B_{A} \frac{\stackrel{\circ}{D}^{A}}{\stackrel{\circ}{ }^{3}} \delta D^{3}
$$

and

$$
-8 \int_{W^{*}} B_{k} \delta D^{k}=-8 \int_{W^{*}}\left[\left(B_{3}+B_{A} \frac{\stackrel{\circ}{D}^{A}}{\stackrel{\circ}{ }^{3}}\right) \delta D^{3}-\left(\frac{\stackrel{\circ}{D}^{3}}{\sigma} B_{A} \varepsilon^{A B}\right)_{, B} \chi\right],
$$

where $B_{k}$ stands for $B_{k}(f, \stackrel{\circ}{\Phi})$. Now $\delta D^{3}$ and $\chi$ describe independent degrees of freedom and can be chosen freely. The second term in the last integral (the response to $\chi$ ) vanishes for any $f$ of interest here because of the axial symmetry of the unperturbed metric (both $f$ and $g$ in (40) do not depend upon the variable $\varphi$ ). As $\stackrel{\circ}{D}^{A}=0$, the response to $\delta D^{3}$ is simply $B_{3}$. We want to show that it is impossible to find such Lagrange multipliers $\nu_{1}, \nu_{2}$ that $B_{3}\left(f, \Phi_{m}\right)=B_{3}\left(\Phi_{m}-\nu_{1} \widehat{m}_{H_{1}}-\nu_{2} \widehat{m}_{H_{2}}, \Phi_{m}\right)$ is identically zero, i.e. that $B_{3}\left(\Phi_{m}, \Phi_{m}\right)$, $B_{3}\left(\widehat{m}_{H_{1}}, \Phi_{m}\right)$ and $B_{3}\left(\widehat{m}_{H_{2}}, \Phi_{m}\right)$ are linearly independent. We are unable to derive analytic formulae for $\widehat{m}_{H_{i}}$, but it is a matter of simple calculations to prove numerically this independence. For this purpose we approximate $\Phi_{m}$ by truncating the series defining its value (equation (52) in case of equal-mass data). Then we calculate the boundary conditions for $\widehat{m}_{H_{i}}$ at $H_{1}$ and $H_{2}\left(\mu= \pm \mu_{0}\right.$ for equal-mass data), find $\widehat{m}_{H_{i}}$ by solving equation $(\Delta-1 / 4) \widehat{m}_{H_{i}}=0$, and finally calculate $B_{3}\left(\Phi_{m}, \Phi_{m}\right)$ and $B_{3}\left(\widehat{m}_{H_{i}}, \Phi_{m}\right)$. The result shows that, indeed, $B_{3}\left(\Phi_{m}, \Phi_{m}\right), B_{3}\left(\widehat{m}_{H_{1}}, \Phi_{m}\right)$ and $B_{3}\left(\widehat{m}_{H_{2}}, \Phi_{m}\right)$ are linearly independent. This proves that the Misner data do not minimize the ADM mass. 


\section{Variations of generic initial data}

The analysis of the Misner data presented in the previous Section shows that to construct

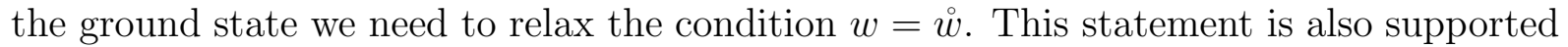
by numerical analysis of two-body data, see [30. In this Section we describe variations of generic initial data, not necessarily conformally flat. For this purpose we consider a generic perturbation $D^{k} \rightarrow D^{k}+\delta D^{k}$ of the field data and calculate the linear part of the corresponding perturbation of the conformal factor: $\Phi \rightarrow \Phi+\delta \Phi$.

To shorten the formulae we will denote $\sqrt{\operatorname{det} w}$ by $\sqrt{w}$. The equations satisfied by $\Phi$ and $\delta \Phi$ on $W^{*}$ take the form:

$$
\begin{gathered}
\left(\Delta_{w}-\frac{\mathcal{R}(w)}{8}\right) \delta \Phi=j[w], \\
\left(\Delta_{w}-\frac{\mathcal{R}(w)}{8}\right) \Phi=0
\end{gathered}
$$

where

$$
j[w]=\frac{1}{8} \Phi \delta \mathcal{R}+\frac{1}{\sqrt{w}}(\delta \sqrt{w}) \Delta_{w} \Phi-\frac{1}{\sqrt{w}} \partial_{i}\left[\left(\delta \sqrt{w} w^{i j}+\sqrt{w} \delta w^{i j}\right) \partial_{j} \Phi\right] .
$$

(Observe that this is a generic form of equation (30).)

We now calculate the coefficients $B_{k}(f, \Phi)$, such that

$$
\int_{W^{*}} \sqrt{w} f\left(\Delta_{w}-\frac{\mathcal{R}(w)}{8}\right) \delta \Phi=\int_{W^{*}} B_{k}(f, \Phi) \delta D^{k} .
$$

We follow the procedure described in Section 4, with obvious modifications. While the expression for $B_{k}$ in case of $w=\stackrel{w}{w}$ were quite simple, in generic case they get rather complicated. For example for $w=\stackrel{w}{w}$ we have $\delta w^{A B}=0$, while in generic case

$$
\delta w^{A B}=\frac{D^{A} \delta D^{B}}{\sigma D^{3}}+\frac{D^{B} \delta D^{A}}{\sigma D^{3}}-\frac{D^{A} D^{B}}{\sigma D^{3}} \frac{\delta D^{3}}{D^{3}},
$$

the other variations get similarly complicated. Here, we give final results for $B_{k}$ (the proofs has been shifted to Appendix (C). It is interesting to compare the formulae given below with equations (40) and (41) which hold for $w=\stackrel{\circ}{\text { (4) }}$. 


$$
\begin{aligned}
& B_{3}=-\frac{1}{2} \frac{\sqrt{w}}{D^{3}} f \Delta_{w} \Phi-\frac{1}{2} \frac{\sqrt{w}}{D^{3}} w^{i j} \Phi_{, i} f_{, j}+\frac{\sqrt{w}}{\sigma} \Phi_{, 3} f_{, 3}-\frac{\sqrt{w}}{\sigma\left(D^{3}\right)^{2}} D^{A} D^{B} \Phi_{, A} f_{, B} \\
& +\frac{1}{8 D^{3}} f \Phi \partial_{i}\left[\sqrt{w}\left(k_{w} M_{w}^{i}+a_{w}^{i}\right)\right] \\
& -\frac{1}{4} f \Phi k_{w, A} D^{A} \frac{1}{\sigma}\left(\frac{\sqrt{w}}{\sigma}\right)^{4}-\frac{1}{8}(f \Phi)_{, i} D^{i} \frac{1}{\sigma}\left(\frac{\sqrt{w}}{\sigma}\right)^{4} k_{w} \\
& +\frac{1}{4} \frac{\sigma}{D^{3}} \frac{D^{A}}{D^{3}}\left[(f \Phi)_{, i} D^{i} \frac{\sqrt{w}}{\sigma^{2}}\right]_{, A}+\frac{1}{8} \frac{\sqrt{w}}{D^{3}} \stackrel{2}{\Delta}(f \Phi)+\frac{1}{8} f \Phi k_{w}^{2} \frac{\sqrt{w}}{D^{3}} \\
& +\frac{1}{8} f \Phi k_{A B} k^{A B} \frac{\sqrt{w}}{D^{3}}-\frac{1}{4}\left[\left(\frac{\sqrt{w}}{\sigma}\right)^{2} f \Phi k^{A B} D^{3}\right]_{, B} \frac{D^{D}}{\left(D^{3}\right)^{2}} \sigma_{A D} \\
& +\frac{1}{8}\left(\frac{\sqrt{w}}{\sigma}\right)^{2} f \Phi k^{A B}\left[\left(\frac{D^{D}}{D^{3}} \sigma_{D A}\right)_{, B}+(\mathrm{A} \leftrightarrow \mathrm{B})\right] \\
& B_{A}=2 \frac{\sqrt{w}}{\sigma} f_{(, 3} \Phi_{, A)}+2 \frac{\sqrt{w} D^{B}}{\sigma D^{3}} f_{(, A} \Phi_{, B)}-\frac{1}{4}\left[(f \Phi)_{, i} D^{i} \frac{\sqrt{w}}{\sigma^{2}}\right]_{, A} \frac{\sigma}{D^{3}} \\
& -\frac{1}{4 D^{3}}\left[\left(\frac{\sqrt{w}}{\sigma}\right)^{2} f \Phi k^{B D} D^{3}\right]_{, B} \sigma_{A D}-\frac{1}{4}\left(\frac{\sqrt{w}}{\sigma}\right)^{2} f \Phi k^{B D} \stackrel{\circ}{\Gamma}_{A B D} \\
& +\frac{1}{4}\left(\frac{\sqrt{w}}{\sigma}\right)^{2} f \Phi k_{w, A}
\end{aligned}
$$

In the above formulae $k_{A B}$ denotes $k_{w A B}$ - the extrinsic curvature tensor of the $\{\mu=$ const. $\}$ leaves with respect to $w, \stackrel{\circ}{\Gamma}_{A B C}$ are Christoffel symbols for the metric $\sigma_{A B}, M_{w}^{i}$ is a normal vector, $M_{w}^{i}=w^{3 i} / \sqrt{w^{33}}$, and ${a_{w}}^{i}:=\frac{1}{2}\left(w^{i j}-M_{w}^{i} M_{w}^{j}\right) \partial_{j} \ln w^{33}$. Note also the relation $\sqrt{w}=\sigma \sqrt{\frac{\sigma}{D^{3}}}$.

\section{Reduction to one degree of freedom}

To analyze the expressions for $B_{k}$ obtained in Section 7 we need first to make some simplifying assumptions. In addition to the previously chosen gauge $w_{A B}=\sigma_{A B}$ we impose the following gauge condition: $k_{w}=0$, i.e. equation (53). As shown in Section 6 the relevant integrand is

$$
\underbrace{\left(B_{3}+B_{A} \frac{D^{A}}{D^{3}}\right)}_{=: B} \delta D^{3}-\left(\frac{D^{3}}{\sigma} B_{A} \varepsilon^{A B}\right)_{, B} \chi .
$$


The degrees of freedom are $\delta D^{3}$ and $\chi$. The response to $\delta D^{3}$, denoted by $B$, is a combination of $B_{3}$ and $B_{A}$, which is simpler than its components, especially if rewritten in terms of $\kappa_{A B}=k_{w A B}-\frac{1}{2} k_{w} \sigma_{A B}$ :

$$
\begin{aligned}
B= & -\frac{1}{2} \frac{\sqrt{w}}{D^{3}} f \Delta_{w} \Phi+\frac{1}{2} \frac{\sqrt{w}}{D^{3}} w^{i j} \Phi_{, i} f_{, j}-\frac{\sqrt{w}}{D^{3}} \sigma^{A B} \Phi_{, A} f_{, B} \\
& +\frac{1}{8 D^{3}} f \Phi \partial_{A}\left(\sqrt{w} a_{w}^{A}\right)+\frac{1}{8}\left(\frac{\sqrt{w}}{\sigma}\right)^{3}{ }^{2} \Delta(f \Phi) \\
& +\frac{1}{8} f \Phi \kappa_{A B} \kappa^{A B} \frac{\sqrt{w}}{D^{3}}-\frac{1}{4}\left[\left(\frac{\sqrt{w}}{\sigma}\right)^{2} f \Phi \kappa^{A B} D^{3}\right]_{, B} \frac{D^{D}}{\left(D^{3}\right)^{2}} \sigma_{A D} \\
& +\frac{1}{8}\left(\frac{\sqrt{w}}{\sigma}\right)^{2} f \Phi \kappa^{A B}\left[\left(\frac{D^{D}}{D^{3}} \sigma_{D A}\right)_{, B}+(\mathrm{A} \leftrightarrow \mathrm{B})\right]_{, B}^{\sigma_{A D}-\frac{D^{A}}{4 D^{3}}\left(\frac{\sqrt{w}}{\sigma}\right)^{2} f \Phi \kappa^{B D} \stackrel{\circ}{A B D}_{A} .}
\end{aligned}
$$

Because of the cylindric symmetry of the problem, it is natural to assume that its solution (the ground state of the two black holes system) also respects this symmetry, i.e. that the functions $D^{k}$ do not depend upon the coordinate $\varphi$. In this case gauge condition (53) together with non-singularity of the two-vector field $D^{A}$ on $S^{2}$ implies $D^{\eta}=0$. Moreover, it is natural to assume the axial symmetry, i.e. symmetry with respect to transformation $\varphi \rightarrow-\varphi$. This implies that $D^{\varphi}=0$ and $B_{\varphi}=0$. Hence, the response to $\chi$ is zero:

$$
\left(\frac{D^{3}}{\sigma} B_{A} \varepsilon^{A B}\right)_{, B}=\left(\frac{D^{3}}{\sigma} B_{\eta} \varepsilon^{\eta \varphi}\right)_{, \varphi}+\left(\frac{D^{3}}{\sigma} B_{\varphi} \varepsilon^{\varphi \eta}\right)_{, \eta}=0
$$

Denote

$$
u(\mu, \eta)=\frac{1}{\sqrt{w^{33}}}
$$

The function $u$ is now the only non-trivial degree of freedom which we take into account. In this simplified situation we can rewrite $\mathcal{R}(w)$ as:

$$
\mathcal{R}(w)=2+\frac{2}{\sqrt{w}} \partial_{A}\left(\sqrt{w} a_{w}^{A}\right)=2-\frac{2}{u} \stackrel{2}{\Delta} u .
$$

The response (65) to $\delta D^{3}$ reduces to a surprisingly simple formula:

$$
B_{3}(f, \Phi)=\frac{u^{3}}{2}\left[u^{-2} \Phi_{, 3} f_{, 3}-\sigma^{A B} \Phi_{, A} f_{, B}+\frac{1}{4}(\stackrel{2}{\Delta}-1)(f \Phi)\right],
$$

and, consequently, we have:

$$
\begin{aligned}
16 \pi \delta m_{A D M} & =-8 \int B_{3}(\Phi, \Phi) \delta D^{3} \\
& =-8 \int \sigma\left[u^{-2}\left(\Phi_{, 3}\right)^{2}-\Phi_{, A} \Phi^{, A}+\frac{1}{4}(\stackrel{2}{\Delta}-1)\left(\Phi^{2}\right)\right] \delta u .
\end{aligned}
$$


Equation (15) for $\Phi$ may now be written as

$$
\partial_{3}\left(u^{-1} \Phi_{, 3}\right)+\left(u \Phi^{\| A}\right)_{\| A}-\frac{1}{4} \Phi(1-\stackrel{2}{\Delta}) u=-4 \pi \sqrt{d} \boldsymbol{\delta}_{0},
$$

where $\|_{A A}$ is a two-dimensional covariant derivative with respect to $w_{A B}=\sigma_{A B}$. Equation (57) for $\delta \Phi$ reduces now to

$$
\begin{aligned}
& \left(\Delta_{w}-\frac{\mathcal{R}(w)}{8}\right) \delta \Phi \\
& \quad=\frac{1}{u}\left(\left(u^{-2} \Phi_{, 3} \delta u\right)_{, 3}-\delta u \stackrel{2}{\Delta} \Phi-\Phi^{, A} \delta u_{, A}+\frac{1}{4} \Phi \delta u-\frac{1}{4} \Phi \stackrel{2}{\Delta} \delta u\right) .
\end{aligned}
$$

\section{Conclusions}

At this point we are able to fulfill the main goal of our paper: to formulate necessary conditions for the "ground state" of gravitational field around two black holes. Using the above (physically well-motivated) reduction to a single degree of freedom, described by the function $u$, the condition may be formulated as follows: the conformal factor $\Phi$ satisfies equation

$$
\partial_{3}\left(u^{-1} \Phi_{, 3}\right)+\left(u \Phi^{\| A}\right)_{\| A}-\frac{1}{4} \Phi(1-\stackrel{2}{\Delta}) u=-4 \pi \sqrt{d} \boldsymbol{\delta}_{0},
$$

on $W=S^{2} \times[-a, b]$ with Neumann boundary conditions at $\mu=-a$ and $\mu=b$. Analogically, $\widehat{m}_{H_{i}}, i=1,2$, satisfy equations

$$
\partial_{3}\left(u^{-1} \widehat{m}_{H_{i}, 3}\right)+\left(u \widehat{m}_{H_{i}}^{\| A}\right)_{\| A}-\frac{1}{4} \widehat{m}_{H_{i}}(1-\stackrel{2}{\Delta}) u=0
$$

with boundary conditions (46). Let us define

$$
f=\Phi-\sum_{i=1}^{2} \nu_{i} \widehat{m}_{H_{i}}
$$

where $\nu_{1}, \nu_{2}$ are Lagrange multipliers. To find the ground state we look for such function $u(\mu, \eta)$ and numbers $\nu_{1}, \nu_{2}$ that $B_{3}(f, \Phi)=0$, where $B_{3}(f, \Phi)$ is given by equation (68). This condition reads:

$$
u^{-2} \Phi_{, 3} f_{, 3}-\sigma^{A B} \Phi_{, A} f_{, B}+\frac{1}{4}(\stackrel{2}{\Delta}-1)(f \Phi)=0 .
$$

We have, therefore, four equations (72)-(74) for four functions: $\left(u, \Phi, \widehat{m}_{H_{i}}\right)$ and two Lagrange multipliers. Once these equations are solved on $W$, the masses of the holes can be read from the conformal factor $\Phi$; the distance parameter is $2 d$. The masses can be 
controlled indirectly because we control the parameters $a$ and $b$. The asymptotic analysis of equation $B_{3}(f, \Phi)=0$ proves that the fall-off conditions for the metric $g$ we imposed at the very beginning are satisfied for the ground state (see Appendix D for detailed calculations). This proves the consistency of our approach.

Because of the high non-linearity of the problem, there is no chance to solve it analytically. We do much hope that an appropriate numerical analysis will allow to find solutions, which would be a good starting point for a perturbational approach.

\section{A Linearization of the Ricci scalar}

To derive a linear deformation of the Ricci scalar we start from the Gauss-Codazzi identity:

$$
\mathcal{R}(w)=R(w)+{k_{w}}^{2}-k_{w A B} k_{w}{ }^{A B}+\frac{2}{\sqrt{\operatorname{det} w}} \partial_{i}\left[\sqrt{\operatorname{det} w}\left(k_{w} M_{w}^{i}+a_{w}{ }^{i}\right)\right]
$$

where $k_{w A B}$ is the extrinsic curvature tensor of the leaves $\{\mu=$ const. $\}, k_{w}$ is its trace, $M_{w}^{k}$ is the normal vector, $M_{w}^{k}=w^{3 k} / \sqrt{w^{33}}$, and $a_{w}{ }^{i}:=\frac{1}{2}\left(w^{i j}-M_{w}^{i} M_{w}^{j}\right) \partial_{j} \ln w^{33}$. We have $w_{A B}=\sigma_{A B}$, hence

$$
\begin{gathered}
R(w)=2 \\
k_{w}=\sigma^{A B} k_{w A B}=-\frac{\sqrt{w^{33}}}{\sigma}\left(\frac{\sigma w^{3 k}}{w^{33}}\right)_{, k} .
\end{gathered}
$$

Inserting the formulae $D^{k}=\stackrel{\circ}{D}^{k}+\delta D^{k}$, we get, in notation of Section 4, the following linear approximations:

$$
\begin{gathered}
k_{w}=-\sqrt{\frac{D^{3}}{\sigma}}\left[\frac{1}{D^{3}} D_{, k}^{k}+\frac{D^{k}}{\sigma}\left(\frac{\sigma}{D^{3}}\right)_{, k}\right] \approx-\frac{1}{\sigma} \delta D_{, A}^{A}, \\
k_{w}{ }^{2}-k_{w A B} k_{w}{ }^{A B} \approx 0, \\
M_{w}^{A}=O\left(\delta D^{k}\right), \quad \stackrel{\circ}{M}_{w}^{3}=1, \quad a_{w}{ }^{3}=0, \quad a_{w}{ }^{A} \approx \frac{1}{2} \sigma^{A B}\left(\frac{\delta D^{3}}{\sigma}\right)_{, B} .
\end{gathered}
$$

Incorporating these into the formula for $\mathcal{R}$ we finally get:

$$
\mathcal{R}(w)-2 \approx-\frac{2}{\sigma}\left(\delta D^{A}\right)_{, A 3}+\frac{1}{\sigma} \partial_{A}\left[\sigma \sigma^{A B}\left(\frac{\delta D^{3}}{\sigma}\right)_{, B}\right] .
$$

\section{B Schwarzschild metric - bispherical foliation}

The metric of the $t=0$ slice for the Schwarzschild solution with the mass $m$ is (we fix the value of the conformal factor $\Psi_{s}$ at infinity to be $\sqrt{d}$ ):

$$
g_{s}=\Psi_{s}^{4} \mathbf{b}=\Psi_{s}^{4}\left(\mathrm{~d} x^{2}+\mathrm{d} y^{2}+\mathrm{d} z^{2}\right), \quad \Psi_{s}=\sqrt{d}+\frac{m}{2 \sqrt{d} \bar{r}},
$$


where $\bar{r}=\sqrt{x^{2}+y^{2}+\left(z-z_{0}\right)^{2}}$. We introduce the bispherical coordinates

$$
x=\frac{\sin \eta \cos \varphi}{\cosh \mu-\cos \eta}, \quad y=\frac{\sin \eta \sin \varphi}{\cosh \mu-\cos \eta}, \quad z=\frac{\sinh \mu}{\cosh \mu-\cos \eta}
$$

and choose such $z_{0}$ that the minimal surface $\bar{r}=\frac{m}{2 d}$ coincides with $\mu=-a$ sphere for some $a>0$. This leads to the following formulae:

$$
\begin{gathered}
z_{0}=-\sqrt{1+\frac{m^{2}}{4 d^{2}}}=\frac{\cosh a}{\sinh a}, \quad a=\operatorname{arsinh} \frac{2 d}{m} \\
\bar{r}^{2}=\left(\frac{\sinh \mu}{\cosh \mu-\cos \eta}+\sqrt{1+\frac{m^{2}}{4 d^{2}}}\right)^{2}+\frac{\sin ^{2} \eta}{(\cosh \mu-\cos \eta)^{2}} \\
\Phi_{s}=\Psi_{s} \sqrt{h}=\sqrt{d} \sqrt{h}+\frac{m}{2 \sqrt{d}} \frac{\sqrt{h}}{\bar{r}} \\
\frac{\sqrt{h}}{\bar{r}}=\left[\cosh \mu+\cos \eta+2 \sqrt{1+\frac{m^{2}}{4 d^{2}}} \sinh \mu+\left(1+\frac{m^{2}}{4 d^{2}}\right)(\cosh \mu-\cos \eta)\right]^{-1 / 2}
\end{gathered}
$$

\section{Proof of formulae (62) and (63)}

In this Appendix we use notation introduced in Section 7. The first step in derivation of (62) and (63) is to express the linearized term (59) (denoted by $j[w]$ ) in terms of $\delta D^{k}$. It is a matter of straightforward calculation to see that

$$
\begin{gathered}
\frac{1}{\sqrt{w}}(\delta \sqrt{w}) \Delta_{w} \Phi=-\frac{1}{2} \frac{\delta D^{3}}{D^{3}} \Delta_{w} \Phi \\
-\frac{1}{\sqrt{w}} \partial_{i}\left[\left(\delta \sqrt{w} w^{i j}+\sqrt{w} \delta w^{i j}\right) \partial_{j} \Phi\right]=\frac{1}{2 \sqrt{w}} \partial_{i}\left(\sqrt{w} \frac{\delta D^{3}}{D^{3}} w^{i j} \partial_{j} \Phi\right) \\
-\frac{1}{\sqrt{w}} \partial_{3}\left(\sqrt{w} \frac{\delta D^{j}}{\sigma} \partial_{j} \Phi\right)-\frac{1}{\sqrt{w}} \partial_{A}\left(\sqrt{w} \frac{\delta D^{A}}{\sigma} \partial_{3} \Phi\right) \\
-\frac{1}{\sqrt{w}} \partial_{A}\left[\frac{\sqrt{w}}{\sigma D^{3}}\left(D^{A} \delta D^{B}+D^{B} \delta D^{A}-D^{A} D^{B} \frac{\delta D^{3}}{D^{3}}\right) \partial_{B} \Phi\right] .
\end{gathered}
$$

The remaining term, $\frac{1}{8} \Phi \delta \mathcal{R}$, is more complicated. As in Appendix $\mathrm{A}$, we start from the Gauss-Codazzi identity (75). The first term in $\delta \mathcal{R}$, namely $2 k_{w} \delta k_{w}$, is calculated from

$$
k_{w}=-\frac{1}{\sigma} \sqrt{\frac{D^{3}}{\sigma}}\left(\frac{\sigma D^{k}}{D^{3}}\right)_{, k},
$$


which leads to

$$
\delta k_{w}=\frac{1}{2} \frac{\delta D^{3}}{D^{3}} k_{w}-\frac{1}{\sqrt{w}}\left[\frac{\sigma D_{, k}^{k}}{\left(D^{3}\right)^{2}} \delta D^{3}-\frac{\sigma}{D^{3}} \delta D_{, k}^{k}-\left(\frac{\sigma}{D^{3}}\right)_{, k} \delta D^{k}+D^{k}\left(\frac{\sigma \delta D^{3}}{\left(D^{3}\right)^{2}}\right)\right] .
$$

To linearize the second term, $k_{w A B} k_{w}^{A B}$, we use the following formula for $k_{w A B}$ :

$$
k_{w A B}=\sqrt{\frac{\sigma}{D^{3}}} \Gamma_{A B}^{3},
$$

where $\Gamma_{j k}^{i}$ are Christoffel symbols of the metric $w$. The expression for $k_{w A B}$ in terms of $D^{k}$ is then

$$
k_{w A B}=\frac{1}{2} \sqrt{\frac{\sigma}{D^{3}}}\left[2 \frac{D^{C}}{\sigma} \stackrel{\circ}{\Gamma}_{C A B}-\frac{D^{3}}{\sigma}\left(\left(\frac{D^{D}}{D^{3}} \sigma_{D A}\right)_{, B}+\left(\frac{D^{D}}{D^{3}} \sigma_{D B}\right)_{, A}\right)\right]
$$

and the linearization can be easily calculated. The last (third) term of $\mathcal{R}$, the divergence, involves $M_{w}^{i}$ and $a_{w}^{i}$. Once these are linearized, the remaining calculations are straightforward. We have

$$
M_{w}^{i}=\frac{w^{3 i}}{\sqrt{w^{33}}}, \quad a_{w}^{3}=0, \quad a_{w}^{A}=-\frac{1}{2} \sigma^{A B}\left(\frac{\sigma}{D^{3}}\right)_{, B} \frac{D^{3}}{\sigma},
$$

hence

$$
\delta M_{w}^{i}=\frac{\sqrt{w}}{\sigma^{2}} \delta D^{i}-\frac{1}{2} \frac{\sqrt{w} D^{i}}{\sigma^{2}} \frac{\delta D^{3}}{D^{3}}
$$

and

$$
\delta a_{w}^{A}=\frac{\sigma^{A B}}{2 \sigma}\left[\left(\frac{\sigma}{D^{3}} \frac{\delta D^{3}}{D^{3}}\right)_{, B} D^{3}-\left(\frac{\sigma}{D^{3}}\right)_{, B} \delta D^{3}\right] .
$$

We have, therefore, rewritten $j[w]$ in terms of $\delta D^{k}$. Once this is done, we may read $B_{k}(f, \Phi)$ from the integrand $\sqrt{w} f j[w]$. For example, take the term

$$
-\int_{W^{*}} f \partial_{3}\left(\sqrt{w} \frac{\delta D^{j}}{\sigma} \partial_{j} \Phi\right)
$$

arising from (78). Integration by parts yields

$$
\int_{W^{*}} \sqrt{w} \frac{\delta D^{j}}{\sigma} \partial_{j} \Phi \partial_{3} f=\int_{W^{*}}\left(\sqrt{w} \partial_{3} \Phi \partial_{3} f \frac{\delta D^{3}}{\sigma}+\sqrt{w} \partial_{A} \Phi \partial_{3} f \frac{\delta D^{A}}{\sigma}\right) .
$$

This gives a contribution to $B_{3}(f, \Phi)$, equal $\frac{\sqrt{w}}{\sigma} \partial_{3} \Phi \partial_{3} f$, and a contribution to $B_{A}(f, \Phi)$, equal $\frac{\sqrt{w}}{\sigma} \partial_{A} \Phi \partial_{3} f$. The contributions to $B_{k}(f, \Phi)$ coming from all the other terms may be calculated in exactly the same way. Finally, after rather tedious but simple calculations, we obtain formulae (62) and (63). 


\section{Asymptotic behaviour of solutions of equation $B_{3}(f, \Phi)=0$}

Equation (74) for $B_{3}$ may be analyzed asymptotically, that means near the point $(\mu=$ $0, \eta=0)$ (corresponding to spatial infinity). We rewrite equation (74) as

$$
B_{3}=\frac{u}{2}\left[\Phi_{, 3} f_{, 3}-u^{2} \sigma^{A B} \Phi_{, A} f_{, B}+\frac{u^{2}}{4}(\stackrel{2}{\Delta}-1)(f \Phi)\right]=0 .
$$

We decompose $\Phi, f$ and $u$ as

$$
\Phi=\sqrt{d} \sqrt{h}+\widetilde{\Phi}, \quad f=\sqrt{d} \sqrt{h}+\widetilde{f}, \quad u=1+\widetilde{u}
$$

where $\widetilde{\Phi}, \widetilde{f}, \widetilde{u}$ are bounded, and use $B_{3}(\sqrt{h}, \sqrt{h})=0$. Hence, vanishing of $B_{3}(f, \Phi)$ is equivalent asymptotically to vanishing of the following quantity:

$$
\begin{aligned}
\frac{2}{u} B_{3}= & u^{2}\left[\frac{1}{4}(\stackrel{2}{\Delta}-1)(\widetilde{\Phi} \widetilde{f})-\widetilde{\Phi}^{, A} \widetilde{f}_{, A}\right]+\sqrt{d} \sqrt{h}_{, 3}\left(\widetilde{\Phi}_{, 3}+\widetilde{f}_{, 3}\right) \\
& -\frac{u^{2}}{2} \sqrt{d} \sqrt{h}^{, A}\left(\widetilde{\Phi}_{, A}+\widetilde{f}_{, A}\right)+\widetilde{\Phi}_{, 3} \widetilde{f}_{, 3}+\frac{u^{2}}{4} \sqrt{d} \sqrt{h}(\stackrel{2}{\Delta}-1)(\widetilde{\Phi}+\widetilde{f}) \\
& -\frac{1}{4} d h\left(2 \widetilde{u}+\widetilde{u}^{2}\right) \\
& +\frac{u^{2}}{4} \sqrt{d}(\widetilde{\Phi}+\widetilde{f}) \stackrel{2}{\Delta} \sqrt{h}-\frac{d}{2}\left(2 \widetilde{u}+\widetilde{u}^{2}\right)\left(\sqrt{h}_{,_{A}} \sqrt{h}^{, A}-\sqrt{h} \stackrel{2}{\Delta}^{h}\right) .
\end{aligned}
$$

Let us expand this expression around $(\mu=0, \eta=0)$. We introduce new coordinates $(\varepsilon, \gamma)$ :

$$
\begin{aligned}
& \mu=-\varepsilon \cos \gamma, \\
& \eta=\varepsilon \sin \gamma
\end{aligned}
$$

The asymptotic formulae

$$
\begin{gathered}
\sqrt{h}=\sqrt{2} \varepsilon^{-1}+O(\varepsilon), \quad \sqrt{h}_{, 3}=+\sqrt{2} \cos \gamma \varepsilon^{-2}+O(1), \\
\sqrt{h}_{, \eta}=-\sqrt{2} \sin \gamma \varepsilon^{-2}+O(1), \\
\stackrel{2}{\Delta} \sqrt{h}=\left(\sqrt{2}-3 \sqrt{2} \cos ^{2} \gamma\right) \varepsilon^{-3}+O\left(\varepsilon^{-1}\right)=-\sqrt{h}_{, 33}
\end{gathered}
$$

and the assumption $\widetilde{u}=O(\varepsilon)$ allow us to extract the lowest power of $\varepsilon$, namely $\varepsilon^{-3}$ from equation (82). The only terms with $\varepsilon^{-3}$ are the last two terms in equation (82) (the last line). Substituting asymptotic expansions we get

$$
\frac{2}{u} B_{3}=\frac{1}{\varepsilon^{3}}\left[\frac{\sqrt{2}}{4} \sqrt{d}(\widetilde{\Phi}+\widetilde{f})\left(1-3 \cos ^{2} \gamma\right)-\frac{4 d \widetilde{u}}{\varepsilon} \cos ^{2} \gamma\right]+O\left(\varepsilon^{-2}\right) .
$$


By assumption $\widetilde{u}$ is a differentiable function of $\mu$ and $\eta$, hence $\widetilde{u}=\varepsilon\left(C_{1} \sin \gamma+C_{2} \cos \gamma\right)+$ $O\left(\varepsilon^{2}\right), C_{1}, C_{2}$ being constant. The functions of $\gamma$ in (87) are linearly independent and the necessary condition for asymptotic vanishing of $B_{3}$ is

$$
\begin{gathered}
\widetilde{u}=O\left(\varepsilon^{2}\right), \\
\widetilde{\Phi}(\mu=0, \eta=0)+\widetilde{f}(\mu=0, \eta=0)=0 .
\end{gathered}
$$

The condition (88) means that the metric $g$ is of the form

$$
g=\Psi^{4}\left(\mathbf{b}+O\left(r^{-2}\right)\right)
$$

in accordance with the asymptotic conditions imposed in Section 2 ,

\section{References}

[1] P. A. M. Dirac, Classical theory of radiating electrons, Proc. Roy. Soc. A 167 (1938), $148-169$.

[2] R. Haag, Die Selbstwechselwirkung des Elektrons, Naturforsch. 10 a (1955), 752-761.

[3] J. Kijowski, Electrodynamics of moving particles, Gen. Relat. Grav. 26 (1994), 167201. See also On electrodynamical self-interaction, Acta Phys. Pol. A 85 (1994), $771-787$.

[4] H. P. Gittel, J. Kijowski, E. Zeidler, The relativistic dynamics of the combined particle-field system in renormalized classical electrodynamics, Commun. Math. Phys. 198 (1998), 711-736.

[5] J. Kijowski and P. Podleś, Born renormalization in classical Maxwell electrodynamics, J. Geom. Phys. 48 (2003), 369-384.

[6] J. Jezierski, Stability of Reissner-Nordström solution with respect to small perturbations of initial data, Classical and Quantum Gravity 11 (1994), 1055-1068.

[7] J. Jezierski, Perturbation of initial data for spherically symmetric charged black hole and Penrose conjecture, Acta Physica Polonica B 25 (1994), 1413-17.

[8] U. Brauer, E. Malec, N. O'Murchadha, Trapped surfaces in spherical expanding open universes, Phys. Rev. D 49 (1994), 5601-5603; E. Malec, N. O'Murchadha, Trapped surfaces and the Penrose inequality in spherically symmetric geometries, Phys. Rev. D 49 (1994), 6931-6934.

[9] G. Huisken and T. Ilmanen, The Inverse Mean Curvature Flow and the Riemannian Penrose Inequality, Journ. Diff. Geometry 59 (2001), 353-437. 
[10] R. Bartnik, Phase space for the Einstein equations, (2004), http://arxiv.org/abs/gr-qc/0402070

[11] D. Sudarsky and R. M. Wald, Extrema of mass, stationarity, and staticity, and solutions to the Einstein Yang-Mills equations, Phys. Rev. D 46 (1992), 1453-1474.

[12] G.L. Bunting and A.K.M. Masood-ul-Alam, Nonexistence of multiple black holes in asymptotically Euclidean static vacuum space-time, Gen. Rel. and Grav. 19 (1987), $147-154$.

[13] D. Giulini, On the construction of time-symmetric black hole initial data, Black Holes: Theory and Observation, Springer Verlag, Berlin, 1998.

[14] C. Misner, Wormhole initial conditions, Phys. Rev. 118 (1960), 1110-1111.

[15] D. Brill and R. Lindquist, Interaction energy in geometrostatics, Phys. Rev. 131 (1963), 471-476.

[16] L.B. Szabados, Quasi-local energy-momentum and angular momentum in GR: a review article, Living Rev. Relativity 7 (2004), 4. [Online article]: cited on 17 Jun 2004, http://www . livingreviews . org/lrr-2004-4

[17] H.L. Bray and P.T. Chruściel, The Penrose inequality, in: The Einstein Equations and the Large Scale Behavior of Gravitational Fields (50 Years of the Cauchy Problem in General Relativity), P.T. Chruściel, H. Friedrich, Eds, Birkhaeuser, Basel, 2004, pp. 39-70, http://arxiv.org/abs/gr-qc/0312047

[18] J. Kijowski On positivity of gravitational energy, in Proceedings of the fourth Marcel Grossmann meeting on General Relativity, Rome, 1985, R. Ruffini - editor, Elsvier Science Publishers B. V., 1986, p. 1681 - 1686.

[19] J. Jezierski and J. Kijowski, Positivity of total energy in general relativity, Physical Review D 36 (1987), 1041-1044.

[20] Sz. Łȩski, Two black hole initial data, Phys. Rev. D 71 (2005), 124018, http://arxiv.org/abs/gr-qc/0502085.

[21] G. Cook, Initial data for axisymmetric black-hole collisions, Phys. Rev. D 44 (1991), 2983-3000.

[22] C. Misner, The method of images in geometrostatics, Ann. Phys. (NY) 24 (1963), $102-117$.

[23] R. Penrose, Gravitational collapse: the role of general relativity, Riv. del Nuovo Cimento 1, (1969) 252-276. 
[24] V. P. Frolov and I. D. Novikov, Black hole physics: basic concepts and new developments, Kluwer Academic Pub, (1998); in Russian: Новиков И. Д., Фролов В. П., Физика черньх дыр, Наука, (Москва 1986).

[25] M. Heusler, Black hole uniqueness theorems, Cambridge lecture notes in physics 6, University Press, p. 119 (Cambridge 1996)

[26] S. Klainerman and F. Nicolo, The evolution problem in general relativity, Progress in mathematical physics, vol. 25, Birkhäuser 2003.

[27] B.S. Kay and R.M. Wald, Linear stability of Schwarzschild under perturbations which are non-vanishing on the bifurcation 2-sphere, Class. Quantum Grav. 4 (1987), 893898.

[28] A.M. Abrahams and R.W. Price, Black-hole collisions from Brill-Lindquist initial data: Predictions of perturbation theory, Phys. Rev. D 53 (1996), 1972, http://arxiv.org/abs/gr-qc/9509020.

[29] L.M. Burko, Towards a wave-extraction method for numerical relativity. V. Extracting the Weyl scalars in the quasi-Kinnersley tetrad from spatial data, Phys. Rev. D 75 (2007), 084039, http://arxiv.org/abs/gr-qc/0701101.

[30] K. Martel and E. Poisson, A one-parameter family of time-symmetric initial data for the radial infall of a particle into a schwarzschild black hole, Phys. Rev. D 66 (2002), 084001. 